\title{
Homeostatic Synaptic Plasticity: Local and Global Mechanisms for Stabilizing Neuronal Function
}

\author{
Gina Turrigiano \\ Department of Biology and Center for Behavioral Genomics, Brandeis University, Waltham, \\ Massachusetts 02493 \\ Correspondence: turrigiano@brandeis.edu
}

\begin{abstract}
Neural circuits must maintain stable function in the face of many plastic challenges, including changes in synapse number and strength, during learning and development. Recent work has shown that these destabilizing influences are counterbalanced by homeostatic plasticity mechanisms that act to stabilize neuronal and circuit activity. One such mechanism is synaptic scaling, which allows neurons to detect changes in their own firing rates through a set of calcium-dependent sensors that then regulate receptor trafficking to increase or decrease the accumulation of glutamate receptors at synaptic sites. Additional homeostatic mechanisms may allow local changes in synaptic activation to generate local synaptic adaptations, and network-wide changes in activity to generate network-wide adjustments in the balance between excitation and inhibition. The signaling pathways underlying these various forms of homeostatic plasticity are currently under intense scrutiny, and although dozens of molecular pathways have now been implicated in homeostatic plasticity, a clear picture of how homeostatic feedback is structured at the molecular level has not yet emerged. On a functional level, neuronal networks likely use this complex set of regulatory mechanisms to achieve homeostasis over a wide range of temporal and spatial scales.
\end{abstract}

$M$ ore than 50 years ago, Walter Cannon marveled that "somehow the unstable stuff of which we are composed has learned the trick of maintaining stability" (Cannon 1932). Along with Claude Bernard, Cannon had realized that complex physiological systems are built in a way that promotes stability, or "homeostasis," of key physiological parameters such as body temperature and blood glucose levels. Recently it has become clear that neuronal activity is itself a key physiological param- eter that is subject to homeostatic regulation. During development billions of neurons wire themselves up into complex networks and manage to reach a state where they can generateand then maintain-stable activity patterns throughout the life of an organism. What makes this so extraordinary is that these circuits are not static, but are constantly undergoing modifications to allow organisms to store information and adapt their behavior to a changing environment. Somehow the forces that generate flexi-

Editors: Morgan Sheng, Bernardo Sabatini, and Thomas Südhof

Additional Perspectives on The Synapse available at www.cshperspectives.org

Copyright (C) 2012 Cold Spring Harbor Laboratory Press; all rights reserved; doi: 10.1101/cshperspect.a005736

Cite this article as Cold Spring Harb Perspect Biol 2012;4:a005736 
G. Turrigiano

bility and those that generate stability are able to coexist without interfering with each other, and together enable the remarkable ability of organisms to generate behaviors that are both coherent and adaptable.

Neural circuits are subject to many forces that work to destabilize their activity. For example, synapse-specific correlation-based plasticity mechanisms such as long-term potentiation (LTP) and depression (LTD) are widely thought to contribute to learning and information storage, but theoreticians have long appreciated that they generate a powerful destabilizing force on network function (Miller and MacKay 1994; Abbott and Nelson 2000). This is because when synapses undergo LTP they are better able to depolarize the postsynaptic neurons, which will increase the probability that they will undergo further LTP-leading to unconstrained synaptic strengthening. A related problem is that as correlated activity drives strengthening of specific synapses, and the postsynaptic neuron is driven more strongly, synapses that initially were only poorly correlated with postsynaptic firing will be better able to fire the postsynaptic neuron, so they too can become strengthened. Thus, without forces that prevent the excitability of the postsynaptic neuron from changing in response to correlation-based plasticity mechanisms, their specificity breaks down and information can no longer be effectively stored through differences in synaptic strengths. Many forms of correlation-based plasticity have now been described biologically, and each is likely to introduce its own unique destabilizing influences on neural circuit function (Abbott and Nelson 2000).

There has been a recent explosion of work into the biological solutions that neurons and networks use to stabilize activity, and collectively these mechanisms have been termed "homeostatic plasticity" (Marder and Prinz 2003; Turrigiano and Nelson 2004; Davis 2006; Turrigiano 2008; Pozo and Goda 2010). To be considered truly homeostatic, a plasticity mechanism should regulate a key parameter (such as average neuronal firing rate) around some set-point value (Fig. 1A). To accomplish this feat, neurons must sense some aspect of "activity," generate an error signal when this deviates from a set point, then use this error signal to change excitability in the correct direction to move activity back toward this set point. Here we will define a homeostatic form of plasticity as one that acts to stabilize the activity of a neuron or neuronal circuit around some set-point value; some mechanisms might be adaptive (in that they tend to oppose instability) without being strictly homeostatic. Currently a number of phenomena have been described that may contribute to stabilization of neuronal activity, including the activity-dependent regulation of intrinsic neuronal firing (Marder and Prinz 2003; Zhang and Linden 2003); pre- and postsynaptic forms of excitatory synaptic plasticity, such as synaptic scaling, that adjust all of a neuron's excitatory synapses up or down in the right direction to stabilize firing (Turrigiano and Nelson 2004; Davis 2006); the balancing of excitation and inhibition within neuronal networks (Maffei et al. 2004; Gonzalez-Islas and Wenner 2006); compensatory changes in synapse number (Kirov et al. 1999; Wierenga et al. 2006); metaplastic mechanisms that adjust the relative ease of inducing LTP and LTD (Bienenstock et al. 1982); and homeostatic regulation of intrinsic excitability (Marder and Goaillard 2006; Turrigiano 2011). Although all of these phenomena are theoretically capable of serving a homeostatic function, for many of them such a function has not been directly shown. In this collection on the synapse, I will focus on synaptic mechanisms that are likely to contribute to network homeostasis.

\section{HOMEOSTATIC PLASTICITY AT THE NEUROMUSCULAR JUNCTION}

The stability problem at the neuromuscular junction (NMJ) is fairly simple: As muscle fibers grow, motor neurons must remain capable of bringing them over threshold for contraction. This matching of motoneuron and muscle fiber properties is accomplished through an active process that requires signaling between motoneuron and muscle, and can keep the gain of neuromuscular coupling remarkably constant. Some of the first reports of homeostatic compensation 


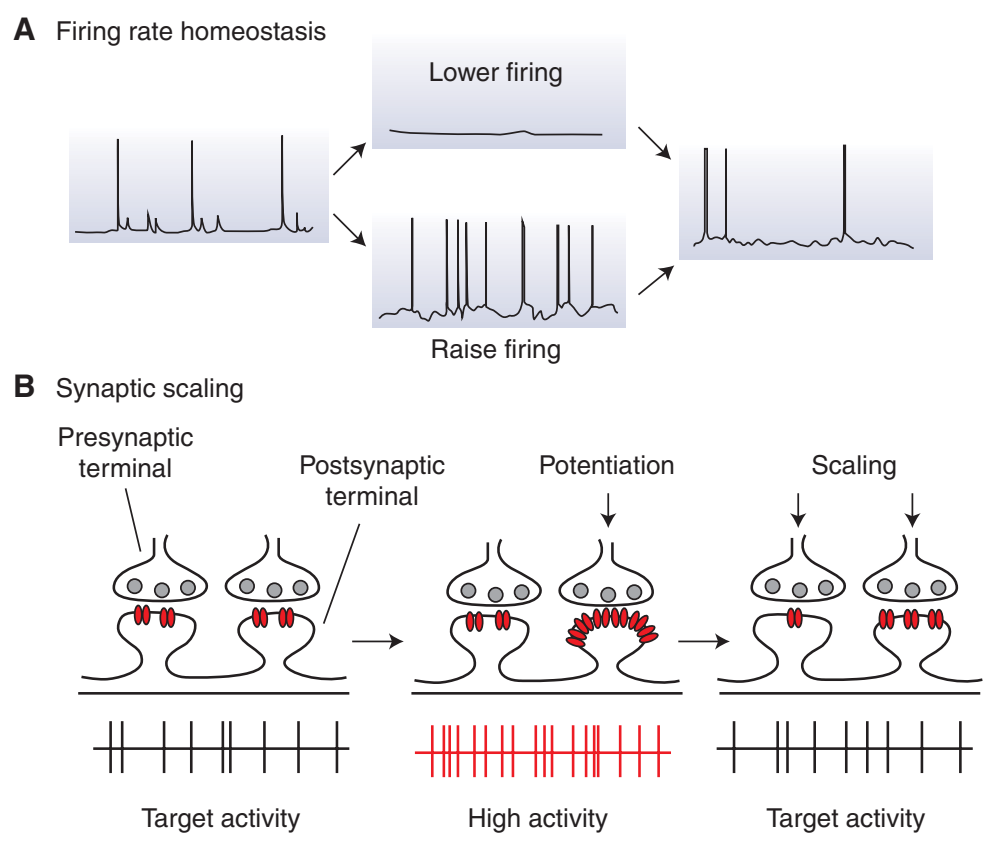

Figure 1. Homeostasis of neuronal firing through homeostatic synaptic plasticity. ( $A$ ) Cartoon illustration of the phenomenon of firing rate homeostasis in dissociated neocortical networks; perturbing firing in either direction results in the homeostatic regulation of synaptic and intrinsic properties so that baseline firing rates are restored. (B) One mechanism contributing to the firing rate homeostasis illustrated in A is synaptic scaling. When activity is perturbed (illustrated here as the potentiation of some inputs through Hebbian mechanisms) this triggers synaptic scaling, which produces a proportional reduction in strength at all synapses of the right magnitude to return firing to baseline levels. Note that, because this mechanism scales synaptic strength up or down proportionally, the relative difference in synaptic strengths induced by Hebbian mechanisms is preserved.

of excitability in the nervous system came from the denervation supersensitivity literature (Berg and Hall, 1975; Sharpless 1975), where it was observed that loss of synaptic innervation initiated an increase in muscle excitability through changes in postsynaptic receptor number and localization. More recently work at the mammalian and invertebrate NMJ has shown that perturbations in postsynaptic function can lead to compensatory changes in presynaptic release, and vice versa (Davis and Bezprozvanny 2001).

In Drosophila the ease of genetic manipulations has made it possible to perturb either the presynaptic or postsynaptic side of the synapse and observe exquisitely precise compensation, so that the gain of transmission remains essentially constant. For example, reductions in glutamate receptor function, or chronic hyper- polarization of the muscle, lead to compensatory increases in transmitter release that restore evoked transmission to control levels (Paradis et al. 2001). The mechanisms underlying this presynaptic form of compensation have recently been worked out in some detail. Compensation can occur very quickly when induced by pharmacological blockade of postsynaptic glutamate receptors and occurs in response to subtle changes in the amplitude of excitatory postsynaptic potentials. A reduction in postsynaptic depolarization generates an as-yet unidentified retrograde signal that leads to enhanced transmitter release (Frank et al. 2006). Other elements of this signaling pathway include a presynaptic Eph receptor, the Eph interacting protein Exn, and activation of the Rho GTPase Cdc42. This signaling pathway converges onto the presynaptic calcium channel Cav2.1 to 
G. Turrigiano

enhance presynaptic calcium influx and thus neurotransmitter release (Frank et al. 2009). A functionally similar mechanism coupling postsynaptic muscle activation with presynaptic regulation of neurotransmitter release has recently been described at the Caenorhabditis elegans NMJ (Simon et al. 2008). It should be noted that there is evidence at the Drosophila NMJ for additional parallel signaling pathways during presynaptic compensation (Frank et al. 2006; Goold and Davis 2007; Frank et al. 2009), as well as mechanisms that can induce postsynaptic compensatory changes in quantal amplitude (Davis and Goodman 1998). This complexity in compensatory mechanisms and signaling pathways is a theme we shall return to when we discuss homeostatic plasticity at central synapses.

\section{HOMEOSTATIC PLASTICITY AT CENTRAL MAMMALIAN SYNAPSES}

There is compelling evidence from experiments in neuronal cultures that central neurons are able to maintain average firing rates around a homeostatic set point. When cortical or hippocampal neurons are induced to fire more than normal, over many hours firing returns to baseline levels, and similarly, if neuronal firing is reduced over time, neurons compensate and again firing is restored (Fig. 1A) (Turrigiano et al. 1998; Burrone et al. 2002). Central neurons are embedded in complex networks composed of many cell types, including both excitatory and inhibitory neurons, and small changes in the balance between excitation and inhibition can have major effects on ongoing activity ( $\mathrm{Nel}$ son and Turrigiano 2008). The ability to compensate for external perturbations and maintain stable firing is thus not trivial, and it is not surprising that a rich variety of homeostatic mechanisms operating over various temporal and spatial scales are likely to contribute to this process. For example, there is evidence for both "global" mechanisms that operate on all of a neuron's synapses (Turrigiano 2008), and "local" mechanisms that act on individual or small groups of synapses (Yu and Goda 2009). Similarly, some forms of homeostatic plasticity occur through presynaptic and others through postsynaptic changes in function (Davis and Bezprozvanny 2001). Which of these mechanisms are engaged will likely depend on how activity is modulated, as well as other factors such as cell type and developmental stage. Below we will review the current state of understanding of the signaling pathways and expression mechanisms of several forms of homeostatic synaptic plasticity at central mammalian synapses.

\section{CELL-AUTONOMOUS, GLOBAL SYNAPTIC SCALING OF EXCITATORY SYNAPSES}

Currently the best understood form of homeostatic plasticity at central excitatory synapses is synaptic scaling. Synaptic scaling was first identified in cultured neocortical neurons, where it was observed that perturbing network activity generated compensatory changes in synaptic strength that were in the right direction to restore average firing rates to baseline values (Turrigiano et al. 1998). Pharmacological manipulations of activity are able to induce bidirectional compensatory changes in the unit strength of glutamatergic synapses, which can be measured by recording miniature excitatory postsynaptic currents (mEPSCs, or "minis"). Minis represent the postsynaptic response to release of individual vesicles of neurotransmitters; by measuring minis arising from many synapses onto the same neuron, it was observed that modulating network activity induced uniform increases or decreases in the entire mini amplitude distribution, in effect scaling postsynaptic strength up or down (Turrigiano et al. 1998; Desai et al. 2002; Gainey et al. 2009). Interestingly, synaptic scaling protocols homeostatically regulate both the NMDA and the AMPA component of glutamatergic synaptic currents, and the change in AMPA and NMDA currents are proportional at individual synapses (Watt et al. 2000; Perez-Otano and Ehlers 2005). These changes in mini amplitude translate into changes in the amplitude of evoked transmission, with little or no change in short-term synaptic dynamics (Watt et al. 2000; Maffei et al. 2004; Wierenga et al. 2005). Such a postsynaptic 
scaling process has the attractive property of allowing neurons to stabilize activity without changing the relative strength of synaptic inputs, thus avoiding disrupting information storage or processing mechanisms that rely on differences in synaptic weights (Fig. 1B). Synaptic scaling has now been shown in a variety of central neurons both in vitro and in vivo, including neocortical and hippocampal pyramidal neurons and spinal neurons (O'Brien et al. 1998; Turrigiano et al. 1998; Desai et al. 2002; Stellwagen and Malenka 2006; Goel and Lee 2007; Kim and Tsien 2008; Knogler et al. 2010). Currently, most of the mechanistic work on synaptic scaling has involved neocortical or hippocampal pyramidal neurons in dissociated culture; whether the mechanisms that underlie synaptic scaling in vivo and in other brain regions are similar or distinct to those for cultured cortical neurons remains an open question.

Perturbations in network activity could be sensed by individual neurons as changes in their own firing, local changes in receptor activation, or changes in release of secreted factors. Although some forms of homeostatic plasticity appear to be triggered by local signaling or signaling through secreted factors (these will be reviewed in turn below), there is strong evidence that synaptic scaling is a cell-autonomous process induced by changes in a neuron's own firing. Selectively blocking firing by microperfusion of TTX to the soma of individual neurons scales up synaptic strengths to the same degree as blockade of network activity, whereas local block of synaptic transmission does not induce a local enhancement of receptor accumulation (Ibata et al. 2008). Interestingly, chronic hyperpolarization of an entire neuron by expression of an inwardly rectifying $\mathrm{K}$ channel $\left(\mathrm{K}_{\mathrm{ir}}\right)$ does not induce postsynaptic scaling (Burrone et al. 2002; Hou et al. 2008), suggesting that chronic global hyperpolarization of neuronal dendrites and somata overrides the signals that induce scaling up in response to a drop in firing.

Selectively blocking postsynaptic firing in neocortical neurons scales synapses up through a process that requires a drop in somatic calcium influx, reduced activation of CaMKIV, and transcription (Ibata et al. 2008). This sig- naling pathway then leads to enhanced accumulation of AMPA-type glutamate receptors (AMPAR) in the postsynaptic membrane at all excitatory synapses, thus scaling up mini amplitude and enhancing evoked transmission. This global enhancement of AMPAR abundance in response to activity blockade requires sequences on the carboxyl terminus of the GluA2 subunit of the AMPAR (Gainey et al. 2009), and the majority of studies that have blocked activity with tetrodotoxin (TTX) or AMPAR antagonists have observed a coordinated increase in GluA1 and GluA2 (O'Brien et al. 1998; Wierenga et al. 2005; Cingolani et al. 2008; Sun and Wolf 2009; Anggono et al. 2011; but see Thiagarajan et al. 2005). Additionally, there is evidence that the neurotrophin brain-derived neurotrophic factor (BDNF) (Rutherford et al. 1998), the immediate early gene Arc (Shepherd et al. 2006), the cytokine TNF $\alpha$ (Stellwagen and Malenka 2006; Steinmetz and Turrigiano, 2010), the immune molecule MHC1 (Goddard et al. 2007), $\beta 3$ integrins (Cingolani et al. 2008), the AMPAR binding protein PICK1 (Anggono et al. 2011), and the scaffold proteins PSD-95 and PSD-93 (Sun and Turrigiano 2011) can all contribute to, or are essential for, synaptic scaling in dissociated cultures. Several of these molecules are known to regulate AMPA receptor trafficking; for example, Arc interacts with the endocytic machinery that removes AMPAR from the membrane (Chowdhury et al. 2006), TNF $\alpha$ directly increases synaptic AMPAR accumulation (Beattie et al. 2002; Stellwagen et al. 2005), $\beta 3$ integrins regulate AMPAR surface expression (Cingolani et al. 2008), PICK1 regulates the pool size of intracellular receptors (Anggono et al. 2011), and PSD-95 stabilizes AMPAR at synaptic sites (Bats et al. 2007); but how these various players cooperate to accomplish the homeostatic regulation of AMPAR abundance remains unclear.

Like scaling up, scaling down in response to elevated network activity is a cell-autonomous function of postsynaptic firing, and involves enhanced calcium influx (Fig. 2), gene transcription, the CaMKK/CaMKIV signaling pathway, and targets the GluA2 subunit (Goold and Nicoll 2010). Another calcium-dependent 


\section{G. Turrigiano}

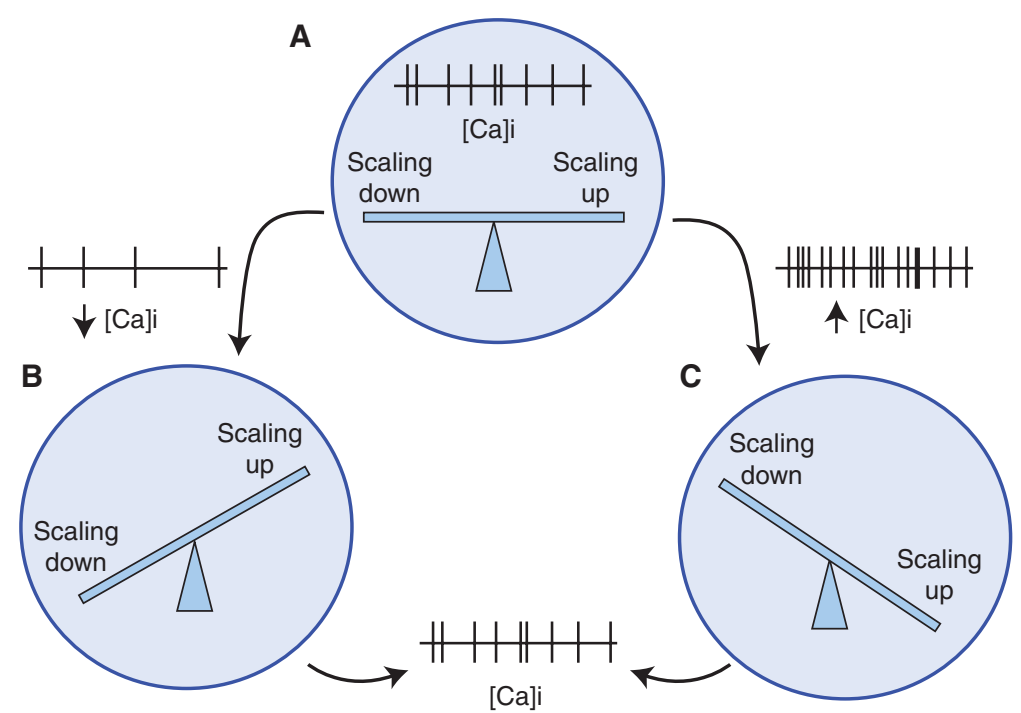

Figure 2. Calcium-dependent pathways regulate both scaling up and scaling down. (A) At a particular average level of somatic calcium influx, scaling up and scaling down will balance each other, and the resulting synaptic equilibrium will help determine the firing rate set point of the neuron. (B) If activity decreases (owing to sensory deprivation, learning-induced LTD, or other factors) then average somatic calcium will also decrease; this will enhance scaling up and reduce scaling down and restore firing to baseline. $(C)$ Conversely, if firing increases and average somatic calcium increases, this will enhance scaling down and reduce scaling up, again restoring firing to baseline.

pathway implicated in scaling down is the pololike kinase 2 (Plk2)-CDK5 signaling pathway (Seeburg et al. 2008). Calcium influx activates Plk2, which when primed by CDK5 can bind to the scaffold protein Spar and trigger its degradation, and this pathway is necessary for the reduction in synaptic AMPAR accumulation induced by elevated activity (Seeburg and Sheng 2008; Seeburg et al. 2008). Plk2 can also reduce surface AMPAR number through a kinaseindependent association with NSF (Evers et al. 2011), suggesting Plk2 may operate through parallel pathways to reduce synaptic strength in response to elevated activity. Two additional calcium-dependent pathways have recently been identified. One involves the immediate early gene Homerla, which is induced in a calcium-dependent manner by enhanced activity and is required for scaling down through a pathway that requires agonist-independent regulation of mGluRs and reduced tyrosine phosphorylation of GluA2 (Hu et al. 2010). The second involves the activation of Eph4A by elevated activity; Eph4A is also necessary for scaling down, and is thought to act by regulating the ubiquitin pathway to control AMPAR degradation (Fu et al. 2011). Thus, raising network firing activates a slew of calcium-dependent signaling pathways, each with elements that are required for the expression of scaling down. One explanation for why so many apparently independent calcium-activated signaling pathways are all necessary for the expression of scaling down is that these signals converge at some point in the scaling pathway to coordinately regulate a critical step in synaptic AMPAR reduction. Alternatively, there may be multiple steps in the scaling pathway that must all be regulated to induce scaling, each of which is targeted by a separate calcium-dependent signaling pathway.

There is conflicting evidence as to whether scaling up and down involve reciprocal modulation of the same signaling pathways. Although scaling up and down have in common reciprocal modulation of somatic calcium influx 
(Fig. 2), and both involve changes in signaling through the CaMKK/CaMKIV pathways (Ibata et al. 2008; Goold and Nicoll 2010), several other signaling and trafficking elements known to be important or essential for scaling up are dispensable for scaling down, including BDNF, TNF $\alpha$, Arc, and PICK1 (Rutherford et al. 1998; Leslie et al. 2001; Shepherd et al. 2006; Stellwagen and Malenka 2006). Further, whereas scaling up is completely dependent on the scaffold protein PSD-95, scaling down can be supported by either PSD-95 or PSD-93 (Sun and Turrigiano 2011). Given the differential dependence of scaling up and down on various aspects of the synaptic regulatory machinery, it seems likely that the signaling pathways involved diverge shortly after the common trigger of altered calcium influx. This suggests that a drop in somatic calcium influx will simultaneously enhance signaling in the pathways that scale synapses up and reduce signaling in the pathways that scale synapses down, and vice versa when firing rises. The homeostatic control of quantal amplitude is thus likely to work in a push-pull manner, with opposing signaling pathways acting to scale synapses up or down. One interesting implication of this model is that the firing rate set point is an emergent property that represents the stable point of all the opposing signaling pathways that operate on synaptic strength (Turrigiano 2008).

As the above discussion should have made abundantly clear, the molecular events that lead from cell-autonomous changes in firing to changes in synaptic AMPAR abundance are incompletely understood. As for homeostatic regulation at the NMJ, it seems that number of signaling pathways are activated by synaptic scaling protocols, and dozens of molecules have been identified that contribute in some way to the ability of synapses to adaptively enhance or reduce receptor accumulation. Although some of these players are undoubtedly integral signaling elements for scaling, others will instead turn out to be permissive, either because they act constitutively to maintain synapses in a plastic state (for example, $\mathrm{TNF} \alpha$ ) (Steinmetz and Turrigiano 2010), or because they are part of the constitutive trafficking pathways that maintain synaptic receptor abundance.

Interestingly, the dependence of synaptic scaling on GluA2 differentiates it from other forms of synaptic enhancement, such as local homeostatic plasticity induced by concurrent TTX and $N$-methyl-D-aspartate receptor (NMDAR) block (see below), and many forms of LTP that require regulatory sequences on the GluA1 rather than the GluA2 subunit (Malenka and Bear 2004). Several recent studies have now reinforced the idea that the trafficking mechanisms that underlie enhanced or reduced AMPAR accumulation during homeostatic plasticity are fundamentally different from those that underlie Hebbian forms of plasticity such as LTP and LTD. For example, whereas LTD depends on the SH3-GK domains of PSD-95 (Xu et al. 2008), scaling down depends on the PDZ1/2 domains (which interact with transmembrane AMPA receptor regulatory proteins [TARPs]) (Sun and Turrigiano 2011). Further, enhancement of synaptic strength during LTP and scaling up are differentially dependent on PSD-95; KD does not block LTP but does block scaling up, whereas overexpression occludes LTP but not scaling up (Stein et al. 2003; Ehrlich and Malinow 2004; Ehrlich et al. 2007; Sun and Turrigiano 2011). Similarly, whereas several forms of LTD depend on PICK1 (which binds to GluA2-containing AMPAR) (Steinberg et al. 2006; Terashima et al. 2008; Volk et al. 2010), scaling down is unaffected by loss of PICK1 (Anggono et al. 2011). Thus, cell-autonomously induced synaptic scaling is fundamentally different from LTP and LTD: it operates over a longer temporal scale (hours), a wider spatial scale (global), and utilizes distinct trafficking steps and molecular machinery to enhance or reduce the synaptic accumulation of GluA2-containing AMPAR at most if not all synapses onto a neuron.

It is widely agreed that synaptic scaling involves postsynaptic changes in receptor accumulation (Turrigiano 2008), but under some circumstances additional presynaptic changes can also be recruited (Davis and Bezprozvanny 2001; Rich and Wenner 2007; Thiagarajan 
et al. 2007). For example, there is evidence that glutamate transporter (VGlut) expression in the presynaptic terminal can be enhanced by activity blockade (De Gois et al. 2005; Erickson et al. 2006), raising the possibility that in addition to adding more postsynaptic receptors, neurons might also package more neurotransmitters into each presynaptic vesicle; these two processes would then collaborate to scale up mini amplitude. It is worth noting, however, that postsynaptic knockdown of GluA2 is able to completely block synaptic scaling (Gainey et al. 2009), suggesting that if there is a presynaptic change in transmitter packaging it is not sufficient to enhance mini amplitude on its own. Thus the physiological significance of changes in presynaptic VGlut remain to be determined.

Interestingly, the same manipulation (activity blockade using TTX or AMPAR antagonists) that only affects mini amplitude in young cultures (Turrigiano et al. 1998; Wierenga et al. 2005) can also induce changes in mini frequency and in presynaptic release probability in older neuronal cultures (Wierenga et al. 2006), through a process that involves postsynaptic hyperpolarization and a drop in calcium influx (Burrone et al. 2002; Thiagarajan et al. 2005); why this transition occurs with time in culture is not currently known. Like synaptic scaling, this presynaptic form of plasticity can also be induced in a cell-autonomous manner by a reduction in postsynaptic depolarization (Burrone et al. 2002). The locus of change during homeostatic plasticity has important consequences for circuit function, because (for example) presynaptic changes in release probability will strongly affect short-term plasticity and thus the dynamics of information transfer across synapses, whereas a postsynaptic change in receptor accumulation can scale postsynaptic responsiveness without affecting the short-term dynamics of synaptic transmission (Abbott and Nelson 2000). Given that the postsynaptic and presynaptic responses to activity deprivation can occur independently (Wierenga et al. 2005, 2006), it is likely that they represent distinct forms of plasticity induced by distinct signaling pathways.

\section{LOCAL AND QUASI-LOCAL FORMS OF HOMEOSTATIC SYNAPTIC PLASTICITY IN CENTRAL NEURONS}

In addition to forms of homeostatic plasticity such as synaptic scaling that are induced in a global manner as a function of postsynaptic firing, there is also evidence that local or quasilocal changes in synaptic signaling can induce homeostatic changes in synaptic strength. A truly local form of plasticity would be induced at an individual synapse as a function of changes in presynaptic release and/or postsynaptic receptor activation at an individual synaptic site. Several studies have now looked at the effects of local changes in signaling on postsynaptic receptor accumulation, with mixed results. Lowering presynaptic firing with an inwardly rectifying $\mathrm{K}_{\mathrm{ir}}$ channel was observed to selectively enhance the accumulation of GluA1 at postsynaptic sites, suggesting the existence of a synapse-specific form of homeostatic compensation to reduced receptor activation (Hou et al. 2008). In contrast, three other studies failed to observe such a local enhancement of AMPAR accumulation at inactivated synapses. Local perfusion of a small number of synaptic sites with either TTX to block presynaptic spikes or DNQX/APV to block postsynaptic glutamate receptors failed to enhance postsynaptic AMPAR accumulation (Ibata et al. 2008), as did chronic block of release at a subset of synapses by presynaptic expression of tetanus toxin (Harms and Craig 2005; Ehlers et al. 2007). The ability of synapses to undergo postsynaptic homeostatic regulation as a function of purely local changes in transmitter release and receptor activation thus remains controversial and may require either extreme presynaptic hyperpolarization (such as would be obtained with presynaptic expression of $\mathrm{K}_{\mathrm{ir}}$ ) or a situation in which spiking and transmitter release are both severely impaired (see discussion below on the effects of simultaneous application of TTX and glutamate receptor blockers).

On a theoretical level it is unclear what purpose truly local homeostatic regulation would serve, as potentiating a synapse through an LTPlike mechanism would then lead to a homeostatic 
reduction in strength (and LTD would lead to potentiation), in effect erasing memory storage. On the other hand, theoretical work has suggested that quasi-local forms of homeostasis that act on groups of nearby synapses can serve a useful normalization function without severely disrupting Hebbian plasticity (Rabinowitch and Segev 2006a,b). Thus, it will be important to understand exactly how local "local" homeostatic mechanisms are. Further, it will be important to understand if this mechanism only operates under extreme conditions (such as when presynaptic firing and transmitter release are drastically reduced), or truly acts homeostatically to keep net synaptic activation constant through graded adjustments in postsynaptic strength to compensate for changes in presynaptic release.

Interestingly, when global block of firing with TTX is combined with local glutamate receptor block, there is a local enhancement of GluA1 accumulation at the blocked synapses (Sutton et al. 2006). This enhancement occurs via a fundamentally different mechanism than the global synaptic scaling described above. Enhanced AMPAR accumulation under conditions of both action potential and NMDAR block involves local synthesis and insertion of GluA1-containing/GluA2-lacking AMPAR into synapses, so that the synaptic composition of AMPAR is modified (Ju et al. 2004; Sutton et al. 2006; Aoto et al. 2008). This is in contrast to blocking firing alone, which leads to enhancement of minis through increased synaptic accumulation of GluA2-containing AMPAR (see above). It has recently been suggested that the enhanced GluA1 synthesis and accumulation induced by TTX + APV is mediated through production of retinoic acid (RA) and activation of the RA receptor $\operatorname{RAR} \alpha$, as suppressing RA production or $\mathrm{RAR} \alpha$ receptors blocks the effects of TTX + APV, and RA can stimulate local translation of GluA1 (Aoto et al. 2008; Poon and Chen 2008).

Exactly why and how blocking action potential firing enables local glutamate receptor block to enhance receptor accumulation is not currently known. One outstanding question is whether TTX is acting by blocking presynaptic or postsynaptic firing; although presynaptic block of action potentials coupled to postsynaptic block of NMDAR can trigger enhanced dendritic protein synthesis (Sutton et al. 2007), it is not known whether this combination is also sufficient to trigger enhanced GluA1 synthesis, local synaptic insertion, and enhanced synaptic strength. If presynaptic firing is the relevant signal during this paradigm then one would predict that presynaptic tetanus toxin, which blocks both action potentials and neurotransmitter release in the presynaptic neuron (Ehlers et al. 2007), should trigger local postsynaptic GluA1 accumulation; yet this manipulation does not (Harms and Craig 2005; Ehlers et al. 2007). Clearly there is more to be done to illuminate the pre- and postsynaptic signals and signaling pathways that trigger this form of homeostatic plasticity, as well as its function within neuronal circuits.

On the presynaptic side, there is compelling evidence that homeostatic modulation of neurotransmitter release probability $\left(\mathrm{P}_{\mathrm{r}}\right)$ can happen at the level of individual dendritic branches. Enhanced synaptic activity was observed to reduce $\mathrm{P}_{\mathrm{r}}$ in cultured hippocampal neurons, through a mechanism that was local to particular dendrites (Branco et al. 2008). Synapses onto the same dendrite tended to have similar $\mathrm{P}_{\mathrm{r}}$, and there was an inverse relationship between synapse number and $\mathrm{P}_{\mathrm{r}}$ onto individual dendrites, suggesting that this regulation happens in a "quasi-local" manner as a result of the degree of dendritic depolarization. This is consistent with evidence that chronic hyperpolarization of entire neurons (including dendrites) results in enhanced $\mathrm{P}_{\mathrm{r}}$ (Burrone et al. 2002), suggesting that $P_{r}$ can be bidirectionally regulated by changes in the amount of dendritic depolarization. It is currently somewhat unclear what the induction requirements are for this form of local presynaptic plasticity; local synaptic blockade was not sufficient to enhance $\mathrm{P}_{\mathrm{r}}$ in one study (Branco et al. 2008), whereas in another local synaptic blockadeinduced presynaptic plasticity that was prevented by presynaptic firing (Jakawich et al. 2010); this requirement for presynaptic firing could explain why TTX generally does not 
G. Turrigiano

induce presynaptic homeostatic plasticity (Turrigiano et al. 1998; Wierenga et al. 2005). This quasi-local (dendrite-wide) form of presynaptic homeostasis has been suggested to prevent synaptic saturation owing to strong depolarization from summed inputs onto a dendrite, by reducing $\mathrm{P}_{\mathrm{r}}$ when postsynaptic activation rises too high (Branco et al. 2008). If this mechanism operates in vivo, it predicts an inverse relationship between $\mathrm{P}_{\mathrm{r}}$ and the summed synaptic strength onto a dendritic branch. In apparent conflict with this model, a positive correlation between $\mathrm{P}_{\mathrm{r}}$ and synaptic strength at cortical synapses has been reported (Hardingham et al. 2010; Kay et al. 2011), but because in these studies synaptic strength was measured at unitary connections, it remains an open possibility that such an inverse correlation exists at the level of summed dendritic input.

\section{ROLE OF NETWORK ACTIVITY AND SECRETED FACTORS IN THE INDUCTION OF HOMEOSTATIC PLASTICITY}

In theory homeostatic plasticity mechanisms could also exist at the network level, and operate through the activity-dependent release of secreted factors that act at a number of sites within the network to regulate the excitation/inhibition (E/I) balance. BDNF was the first such secreted factor suggested to play a role in homeostatic plasticity (Rutherford et al. 1997, 1998). BDNF is thought to be released as a function of pyramidal neuron activity, and activates Trk receptors on both pyramidal and interneurons (Lu 2003). Blocking BDNF signaling mimics, and exogenous BDNF application prevents, the effects of activity blockade on excitatory minis, suggesting that in neocortical neurons activitydependent BDNF release could mediate synaptic scaling (Rutherford et al. 1998). However, scaling up can also be induced by chronic changes in the level of depolarization in a BDNF-independent manner (Leslie et al. 2001), and the relationship between BDNF-mediated synaptic scaling and cell-autonomous synaptic scaling of excitatory inputs (Ibata et al. 2008) remains unclear. As mentioned previously, these considerations suggest that there are several distinct signaling pathways that are capable of inducing scaling up of synaptic strengths in response to a drop in activity.

Interestingly, postsynaptic BDNF release has recently been implicated in the rapid local regulation of $\mathrm{P}_{\mathrm{r}}$ (Jakawich et al. 2010), and BDNF is clearly important for the development and regulation of inhibitory synaptic transmission (Huang et al. 1999). A number of reports have suggested that activity-dependent BDNF release is critical for scaling of inhibitory synapses onto pyramidal neurons as well as the homeostatic regulation of excitatory synapses onto inhibitory neurons (Rutherford et al. 1997, 1998; Copi et al. 2005; Swanwick et al. 2006). Consistent with the idea that inhibitory scaling might result from release of a secreted factor during changes in network activity, scaling of inhibitory synapses cannot be induced by hyperpolarization of individual presynaptic or postsynaptic neurons (Hartman et al. 2006). The ability of secreted BDNF to regulate many synapse types both pre- and postsynaptically in a coordinated homeostatic manner suggests that activity-dependent BDNF release contributes to a form of network-wide homeostatic balancing of excitation and inhibition.

Another secreted factor suggested to contribute to homeostatic plasticity is TNF $\alpha$. TNF $\alpha$ is a cytokine that is part of the inflammatory response to pathological states (Bessis et al. 2007). It was recently proposed that prolonged activity blockade (with TTX) increases glial release of TNF $\alpha$, which then acts on neurons to enhance AMPAR insertion and scale up mEPSC amplitude (Stellwagen and Malenka 2006). This model is based on the observations that TNF $\alpha$ levels are elevated by prolonged ( $48 \mathrm{~h}$ ) activity blockade, acute application of TNF $\alpha$ (or conditioned media from activity-blocked cultures) increases mEPSC amplitude, and scaling up in response to prolonged activity blockade is prevented by blocking TNF $\alpha$ signaling (Beattie et al. 2002; Stellwagen et al. 2005; Stellwagen and Malenka 2006). Interestingly, the TNF $\alpha$ involved in scaling up synaptic strengths in response to TTX originates from glia rather than neurons, because wild-type neurons grown on $\mathrm{TNF}^{-/-}$glia did not scale up synaptic 
strengths in response to $48 \mathrm{~h}$ of TTX treatment, whereas $\mathrm{TNF}^{-1-}$ neurons grown on wild-type glia did (Stellwagen and Malenka 2006).

Inconsistent with the above model is the observation that scaling is a gradual and cumulative process evident after as little as $4-6 \mathrm{~h}$ of activity blockade (Sutton et al. 2006; Ibata et al. 2008), whereas $\mathrm{TNF} \alpha$-dependent scaling was only observed after prolonged activity block (48 h) (Stellwagen and Malenka 2006). This raises the possibility that the early phase of scaling is not mediated by $\mathrm{TNF} \alpha$, or alternatively, that $\mathrm{TNF} \alpha$ is permissive rather than instructive for scaling. A recent study suggests that the later interpretation is the correct one. TNF $\alpha$ signaling was not necessary for the induction of early (6 h) scaling, but became essential for maintenance of scaling during prolonged $(>24 \mathrm{~h})$ activity blockade. Further, blocking TNF $\alpha$ signaling for $24 \mathrm{~h}$ before blocking activity with TTX, prevented both early and late scaling (Steinmetz and Turrigiano 2010). TNF $\alpha$ and TTX also appear to influence AMPAR accumulation via distinct mechanisms. Unlike TTX treatment, acute TNF $\alpha$ application does not scale up mini amplitude and adding TNF $\alpha$ to prescaled synapses actually reduces synaptic strength, indicating that scaling and $\mathrm{TNF} \alpha$ are neither additive nor simply occlusive (Steinmetz and Turrigiano 2010). Taken together these studies suggest that glial-derived TNF $\alpha$ release is critical for maintaining synapses in a plastic state in which homeostatic synaptic scaling can be expressed.

\section{SPECIFICITY OF SYNAPTIC SCALING RULES FOR SYNAPSE TYPE}

Neural circuits are composed of many excitatory and inhibitory cell types interconnected in highly specific ways. Thus, you might expect that to stabilize the activity of a neural circuit you would need homeostatic plasticity rules that are specific for particular classes of synapses. In dissociated cortical cultures it has been shown that excitatory synapses onto excitatory pyramidal neurons are scaled up by activity blockade, whereas excitatory synapses onto GABAergic interneurons are not (Rutherford et al.
1998). On the other hand, enhancing network activity does increase excitatory transmission onto GABAergic interneurons (Rutherford et al. 1998; Chang et al. 2010), which should promote the recruitment of additional inhibition when activity in a circuit increases. Further, it has been shown both in vitro and in vivo that inhibitory synapses onto pyramidal neurons are regulated in the opposite direction from excitatory synapses in response to a drop in activity or sensory drive (Kilman et al. 2002; Vale and Sanes 2002; Maffei et al. 2004; Hartman et al. 2006; Huupponen et al. 2007). Interestingly, both in vitro and in vivo studies have documented that homeostatic regulation of inhibition involves coordinated changes in postsynaptic strength, synapse number, and likely presynaptic release probability (Kilman et al. 2002; Maffei et al. 2004; Hartman et al. 2006). These distinct plasticity rules at different classes of excitatory and inhibitory synapse are consistent with an overall homeostatic shift in the balance between excitation and inhibition, as the net effect of the changes induced by activity blockade are to enhance excitation and reduce inhibition, which will act to restore network excitability.

Although in neocortical networks the net effects of changes in excitation and inhibition appear to be homeostatic (Rutherford et al. 1998; Turrigiano et al. 1998; Maffei et al. 2004), different classes of inhibitory synapse are regulated differently by lowered activity. When sensory drive to primary visual cortex is lowered in vivo, connections from fast-spiking basket cells onto layer 4 pyramidal neurons are reduced in amplitude, whereas connections from another class of interneuron become sparser but stronger (Maffei et al. 2004). Similarly, activity blockade with TTX in neocortical slice cultures differentially regulates different classes of inhibitory synapses (Bartley et al. 2008). Further, in hippocampal circuits activity blockade has revealed that under some conditions inhibition can change in the same direction as excitation (Echegoyen et al. 2007), but whether this acts to enhance or oppose stability is not entirely clear.

Interestingly, not all excitatory neurons in cortical networks express synaptic scaling. In 
G. Turrigiano

hippocampal networks CA1 neurons scale synapses up in response to activity blockade, whereas CA3 neurons do not (Kim and Tsien 2008). In visual cortex the expression of synaptic scaling is developmentally regulated (Desai et al. 2002; Maffei et al. 2004, 2006; Maffei and Turrigiano 2008); synaptic scaling is expressed by pyramidal neurons in layer 4 early in postnatal development, but then turns off in layer 4 and begins to be expressed in layer $2 / 3$ pyramidal neurons around the opening of the classical visual system critical period (Desai et al. 2002; Maffei and Turrigiano 2008), where it persists into adulthood (Goel and Lee 2007). An issue that is not yet resolved is whether postsynaptic neurons can differentiate between different types of synapses arising from different sources, and selectively scale one type up or down while leaving others unaffected. This possibility is raised by the finding that the same visual deprivation paradigm that scales minis up in younger mice produces nonmultiplicative changes in minis in older mice, suggesting that not all synapses are affected equally (Goel and Lee 2007); however, this could also reflect the simultaneous activation of synaptic scaling and Hebbian, synapse-specific mechanisms (see Turrigiano 2011 for a discussion of difficulties in interpreting scaling data). Interestingly, a recent study on sensory inputs to Xenopus tectum found that, in multisensory neurons, sensory deprivation of one modality led to a modality-specific enhancement in synaptic transmission, suggesting that synaptic inputs subserving different modalities can undergo independent homeostatic regulation (Deeg and Aizenman 2011). In general, these studies highlight the point that the forms of homeostatic plasticity present at particular classes of synapse onto particular neuronal types will depend critically on their function within the network.

\section{FUNCTIONS OF HOMEOSTATIC PLASTICITY IN VIVO}

Most work on homeostatic synaptic plasticity has used in vitro systems to probe function and uncover molecular mechanisms, but there is now a growing appreciation that homeostatic plasticity is a vital aspect of in vivo circuit function at many stages of development. For example, during embryonic and early postnatal development, homeostatic mechanisms can ensure that spontaneous activity is present in developing spinal circuits (Gonzalez-Islas and Wenner 2006; Knogler et al. 2010), where such activity is vital for driving proper circuit connectivity (Hanson and Landmesser 2004). Similarly, in visual cortex during the second and third postnatal weeks when synaptogenesis is high, there is an inverse relationship between the frequency and amplitude of mEPSCs onto pyramidal neurons, and this can be prevented by raising animals in the dark (Desai et al. 2002). This suggests that as the number of excitatory synapses increases (therefore increasing mEPSC frequency) and visual drive increases, synaptic strength is reduced through an activity-dependent homeostatic mechanism. Such a mechanism could serve the vital developmental function of matching local microcircuit excitability to the strength of sensory drive.

Interestingly, in visual cortical microcircuits the locus of homeostatic plasticity changes as the circuit matures. Early in postnatal development layer 4 (the first input layer to cortex, and the first layer to mature) responds homeostatically to brief ( 2 days) inactivation of the optic nerve with TTX (Desai et al. 2002; Maffei et al. 2004), whereas later (at the opening of the classical visual system critical period) this homeostatic response turns off in layer 4 and migrates to layers $2 / 3$, where it persists into adulthood (Desai, et al. 2002; Maffei et al. 2006; Goel and Lee 2007; Maffei and Turrigiano 2008; Goel et al. 2011). Exactly why the locus of homeostatic plasticity shifts during development remains a mystery. One highly speculative possibility is that later in development (after thalamocortical inputs are established) homeostasis in neocortical layer 4 in response to sensory deprivation would only serve to amplify noise and so becomes maladaptive. In contrast, because neocortical layers $2 / 3$ receive extensive lateral and feedback connections from other cortical areas, homeostasis in layers $2 / 3$ could serve the useful function in the mature animal of encouraging the takeover of deprived cortical 
territory by cortical regions with intact sensory drive.

A role for homeostatic plasticity has recently been suggested to contribute to ocular dominance plasticity in rodent visual cortex (MrsicFlogel et al. 2007; Kaneko et al. 2008). When one eye is deprived of patterned vision during the classical critical period (using lid suture) there is a change in the ability of the two eyes to drive neurons within the binocular portion of visual cortex: cortical neurons rapidly (within 2 or 3 days) lose responsiveness to the deprived eye, and then more slowly (over 4-6 days) gain responsiveness to the nondeprived eye (Frenkel and Bear 2004; Mrsic-Flogel et al. 2007; Kaneko et al. 2008). Interestingly, binocularly driven neurons maintained a similar average level of combined responsiveness to the two eyes, suggesting that the drop in deprived eye responsiveness was compensated by an increase in nondeprived eye responsiveness (Mrsic-Flogel et al. 2007); a very similar response homeostasis following visual deprivation has been reported at retinotectal synapses (Chandrasekaran et al. 2007). Why visual deprivation using lid suture should produce a delayed homeostatic response in visual cortex, whereas blocking optic nerve activity or placing animals in the dark produces an immediate homeostatic response, remains a mystery.

Currently we know little about the cellular and molecular mechanisms underlying homeostatic plasticity in vivo. Visual deprivation induced by optic nerve block scale ups mEPSC amplitude in vivo as in vitro, and results in insertion of GluA2-containing AMPAR (Desai et al. 2002; Maffei and Turrigiano 2008; Gainey et al. 2009). In contrast, dark rearing increases the abundance of GluA2-lacking receptors and requires GluA1 phosphorylation, suggesting that these two modes of deprivation may induce different forms of compensatory synaptic plasticity (Goel and Lee 2007; Goel et al. 2011). Interestingly, the delayed potentiation in visual cortex following lid suture requires TNF $\alpha$ and Arc signaling (Kaneko et al. 2008; McCurry et al. 2011) as does synaptic scaling in vitro (Shepherd et al. 2006; Stellwagen and Malenka 2006; Steinmetz and Turrigiano 2010); however, both these manipulations likely affect many forms of plasticity, so it still remains to be seen if synaptic scaling contributes to the delayed potentiation induced by prolonged lid suture. Taken together, the findings described above suggest that something very like synaptic scaling operates in vivo in the intact visual cortex, but further studies are needed to verify that what is observed in vivo is mechanistically identical to in vitro synaptic scaling.

It should be remembered that homeostatic responses in vivo often couple scaling of excitatory synapses with the selective modification of inhibitory networks, so that there is an overall rebalancing of excitation and inhibition (Turrigiano and Nelson 2004). This highlights the idea that experience-dependent plasticity is unlikely to be explained by a single form of synaptic plasticity, but rather arises through a complex interplay between many forms of excitatory, inhibitory, and also intrinsic plasticity mechanisms occurring at many sites within the cortical microcircuit (Turrigiano 2011). Understanding experience-dependent plasticity will thus require an integrated understanding of how these various forms of plasticity cooperate to modify microcircuit function. The existence of this rich palette of plasticity mechanisms suggests that cortical microcircuits can respond in a very flexible manner to changes in sensory input. In particular, the existence of many forms of homeostatic plasticity operating on different temporal and spatial scales may ensure that network compensation can be achieved in response to a wide range of sensory perturbations.

\section{CONCLUDING REMARKS}

Neurons and networks use a family of homeostatic synaptic plasticity mechanisms to stabilize firing rates in the face of developmental or learning-induced changes in drive, and this contributes to the ability of central neuronal networks to maintain stable function and enables networks to maintain the specificity of synaptic changes that encode information. From considerable work on the activity sensors, expression mechanisms, and signaling pathways involved, the field is beginning to illuminate 


\section{G. Turrigiano}

how homeostatic negative feedback systems are designed and are able to control various aspects of synaptic function. There appear to be independent mechanisms for regulating presynaptic and postsynaptic strength, and there is growing evidence for independent homeostatic mechanisms operating on global and local spatial scales. As additional phenomena are uncovered that appear to operate in a homeostatic or adaptive manner, it will become increasingly important to begin to unravel and differentiate the roles these mechanisms play in tuning synaptic transmission, and how they influence information flow and storage in the intact central nervous system.

\section{REFERENCES}

Abbott LF, Nelson SB. 2000. Synaptic plasticity: Taming the beast. Nat Neurosci 3: 1178-1183.

Anggono V, Clem RL, Huganir RL. 2011. PICK1 loss of function occludes homeostatic synaptic scaling. J Neurosci 31: 2188-2196.

Aoto J, Nam CI, Poon MM, Ting P, Chen L. 2008. Synaptic signaling by all-trans retinoic acid in homeostatic synaptic plasticity. Neuron 60: 308-320.

Bartley AF, Huang ZJ, Huber KM, Gibson JR. 2008. Differential activity-dependent, homeostatic plasticity of two neocortical inhibitory circuits. J Neurophysiol 100: 1983-1994.

Bats C, Groc L, Choquet D. 2007. The interaction between Stargazin and PSD-95 regulates AMPA receptor surface trafficking. Neuron 53: 719-734.

Beattie EC, Stellwagen D, Morishita W, Bresnahan JC, Ha BK, Von Zastrow M, Beattie MS, Malenka RC. 2002. Control of synaptic strength by glial TNF $\alpha$. Science 295: 2282-2285.

Berg DK, Hall ZW. 1975. Increased extrajunctional acetylcholine sensitivity produced by chronic acetylcholine sensitivity produced by chronic post-synaptic neuromuscular blockade. J Physiol 244: 659-676.

Bessis A, Bechade C, Bernard D, Roumier A. 2007. Microglial control of neuronal death and synaptic properties. Glia 55: 233-238.

Bienenstock EL, Cooper LN, Munro PW. 1982. Theory for the development of neuron selectivity: Orientation specificity and binocular interaction in visual cortex. J Neurosci 2: 32-48.

Branco T, Staras K, Darcy KJ, Goda Y. 2008. Local dendritic activity sets release probability at hippocampal synapses. Neuron 59: 475-485.

Burrone J, O’Byrne M, Murthy VN. 2002. Multiple forms of synaptic plasticity triggered by selective suppression of activity in individual neurons. Nature 420: 414-418.

Cannon WB. 1932. The wisdom of the body. W.W. Norton, New York.
Chandrasekaran AR, Shah RD, Crair MC. 2007. Developmental homeostasis of mouse retinocollicular synapses. J Neurosci 27: 1746-1755.

Chang MC, Park JM, Pelkey KA, Grabenstatter HL, Xu D, Linden DJ, Sutula TP, McBain CJ, Worley PF. 2010. Narp regulates homeostatic scaling of excitatory synapses on parvalbumin-expressing interneurons. Nat Neurosci 13: $1090-1097$.

Chowdhury S, Shepherd JD, Okuno H, Lyford G, Petralia RS, Plath N, Kuhl D, Huganir RL, Worley PF. 2006. Arc/Arg3.1 interacts with the endocytic machinery to regulate AMPA receptor trafficking. Neuron 52: 445-459.

Cingolani LA, Thalhammer A, Yu LM, Catalano M, Ramos T, Colicos MA, Goda Y. 2008. Activity-dependent regulation of synaptic AMPA receptor composition and abundance by $\beta 3$ integrins. Neuron 58: 749-762.

Copi A, Jungling K, Gottmann K. 2005. Activity- and BDNF-induced plasticity of miniature synaptic currents in ES cell-derived neurons integrated in a neocortical network. J Neurophysiol 94: 4538-4543.

Davis GW. 2006. Homeostatic control of neural activity: From phenomenology to molecular design. Annu Rev Neurosci 29: 307-323.

Davis GW, Bezprozvanny I. 2001. Maintaining the stability of neural function: A homeostatic hypothesis. Annu Rev Physiol 63: 847-869.

Davis GW, Goodman CS. 1998. Synapse-specific control of synaptic efficacy at the terminals of a single neuron. Nature 392: 82-86.

Deeg KE, Aizenman CD. 2011. Sensory modality-specific homeostatic plasticity in the developing optic tectum. Nat Neurosci 14: 548-550.

De Gois S, Schafer MK, Defamie N, Chen C, Ricci A, Weihe E, Varoqui H, Erickson JD. 2005. Homeostatic scaling of vesicular glutamate and GABA transporter expression in rat neocortical circuits. J Neurosci 25: 7121-7133.

Desai NS, Cudmore RH, Nelson SB, Turrigiano GG. 2002. Critical periods for experience-dependent synaptic scaling in visual cortex. Nat Neurosci 5: 783-789.

Echegoyen J, Neu A, Graber KD, Soltesz I. 2007. Homeostatic plasticity studied using in vivo hippocampal activity-blockade: Synaptic scaling, intrinsic plasticity and age-dependence. PLoS One 2: e700.

Ehlers MD, Heine M, Groc L, Lee MC, Choquet D. 2007. Diffusional trapping of GluR1 AMPA receptors by inputspecific synaptic activity. Neuron 54: 447-460.

Ehrlich I, Malinow R. 2004. Postsynaptic density 95 controls AMPA receptor incorporation during long-term potentiation and experience-driven synaptic plasticity. J Neurosci 24: 916-927.

Ehrlich I, Klein M, Rumpel S, Malinow R. 2007. PSD-95 is required for activity-driven synapse stabilization. Proc Natl Acad Sci 104: 4176-4181.

Erickson JD, De Gois S, Varoqui H, Schafer MK, Weihe E. 2006. Activity-dependent regulation of vesicular glutamate and GABA transporters: A means to scale quantal size. Neurochem Int 48: 643-649.

Evers DM, Matta JA, Hoe HS, Zarkowsky D, Lee SH, Isaac JT, Pak DT. 2010. Plk2 attachment to NSF induces homeostatic removal of GluA2 during chronic overexcitation. Nat Neurosci 13: 1199-1207. 
Frank CA, Kennedy MJ, Goold CP, Marek KW, Davis GW. 2006. Mechanisms underlying the rapid induction and sustained expression of synaptic homeostasis. Neuron 52: $663-677$.

Frank CA, Pielage J, Davis GW. 2009. A presynaptic homeostatic signaling system composed of the Eph receptor, ephexin, Cdc42, and Cav2.1 calcium channels. Neuron 61: 556-569.

Frenkel MY, Bear MF. 2004. How monocular deprivation shifts ocular dominance in visual cortex of young mice. Neuron 44: 917-923.

Fu AK, Hung KW, Fu WY, Shen C, Chen Y, Xia J, Lai KO, Ip NY. 2011. APC(Cdh1) mediates EphA4-dependent downregulation of AMPA receptors in homeostatic plasticity. Nat Neurosci 14: 181-189.

Gainey MA, Hurvitz-Wolff JR, Lambo ME, Turrigiano GG. 2009. Synaptic scaling requires the GluR2 subunit of the AMPA receptor. J Neurosci 29: 6479-6489.

Goddard CA, Butts DA, Shatz CJ. 2007. Regulation of CNS synapses by neuronal MHC class I. Proc Natl Acad Sci 104: $6828-6833$.

Goel A, Lee HK. 2007. Persistence of experience-induced homeostatic synaptic plasticity through adulthood in superficial layers of mouse visual cortex. J Neurosci 27: 6692-6700.

Goel A, Xu LW, Snyder KP, Song L, Goenaga-Vazquez Y, Megill A, Takamiya K, Huganir RL, Lee HK. 2011. Phosphorylation of AMPA receptors is required for sensory deprivation-induced homeostatic synaptic plasticity. PLoS One 6: e18264.

Gonzalez-Islas C, Wenner P. 2006. Spontaneous network activity in the embryonic spinal cord regulates AMPAergic and GABAergic synaptic strength. Neuron 49: 563-575.

Goold CP, Davis GW. 2007. The BMP ligand Gbb gates the expression of synaptic homeostasis independent of synaptic growth control. Neuron 56: 109-123.

Goold CP, Nicoll RA. 2010. Single-cell optogenetic excitation drives homeostatic synaptic depression. Neuron 68 : 512-528.

Hanson MG, Landmesser LT. 2004. Normal patterns of spontaneous activity are required for correct motor axon guidance and the expression of specific guidance molecules. Neuron 43: 687-701.

Hardingham NR, Read JC, Trevelyan AJ, Nelson JC, Jack JJ, Bannister NJ. 2010. Quantal analysis reveals a functional correlation between presynaptic and postsynaptic efficacy in excitatory connections from rat neocortex. J Neurosci 30: 1441-1451.

Harms KJ, Craig AM. 2005. Synapse composition and organization following chronic activity blockade in cultured hippocampal neurons. J Comp Neurol 490: 72-84.

Hartman KN, Pal SK, Burrone J, Murthy VN. 2006. Activitydependent regulation of inhibitory synaptic transmission in hippocampal neurons. Nat Neurosci 9: 642-649.

Hou Q, Zhang D, Jarzylo L, Huganir RL, Man HY. 2008. Homeostatic regulation of AMPA receptor expression at single hippocampal synapses. Proc Natl Acad Sci 105: 775-780.

Hu JH, Park JM, Park S, Xiao B, Dehoff MH, Kim S, Hayashi T, Schwarz MK, Huganir RL, Seeburg PH, et al. 2010.
Homeostatic scaling requires group I mGluR activation mediated by Homerla. Neuron 68: 1128-1142.

Huang ZJ, Kirkwood A, Pizzorusso T, Porciatti V, Morales B, Bear MF, Maffei L, Tonegawa S. 1999. BDNF regulates the maturation of inhibition and the critical period of plasticity in mouse visual cortex. Cell 98: 739-755.

Huupponen J, Molchanova SM, Taira T, Lauri SE. 2007. Susceptibility for homeostatic plasticity is down-regulated in parallel with maturation of the rat hippocampal synaptic circuitry. J Physiol 581: 505-514.

Ibata K, Sun Q, Turrigiano GG. 2008. Rapid synaptic scaling induced by changes in postsynaptic firing. Neuron 57: 819-826.

Jakawich SK, Nasser HB, Strong MJ, McCartney AJ, Perez AS, Rakesh N, Carruthers CJ, Sutton MA. 2010. Local presynaptic activity gates homeostatic changes in presynaptic function driven by dendritic BDNF synthesis. Neuron 68: 1143-1158.

Ju W, Morishita W, Tsui J, Gaietta G, Deerinck TJ, Adams SR, Garner CC, Tsien RY, Ellisman MH, Malenka RC. 2004. Activity-dependent regulation of dendritic synthesis and trafficking of AMPA receptors. Nat Neurosci 7: 244-53.

Kaneko M, Stellwagen D, Malenka RC, Stryker MP. 2008. Tumor necrosis factor- $\alpha$ mediates one component of competitive, experience-dependent plasticity in developing visual cortex. Neuron 58: 673-680.

Kay L, Humphreys L, Eickholt BJ, Burrone J. 2011. Neuronal activity drives matching of pre- and postsynaptic function during synapse maturation. Nat Neurosci 14: 688 690.

Kilman V, van Rossum MC, Turrigiano GG. 2002. Activity deprivation reduces miniature IPSC amplitude by decreasing the number of postsynaptic $\mathrm{GABA}(\mathrm{A})$ receptors clustered at neocortical synapses. J Neurosci 22: 13281337.

Kim J, Tsien RW. 2008. Synapse-specific adaptations to inactivity in hippocampal circuits achieve homeostatic gain control while dampening network reverberation. Neuron 58: 925-937.

Kirov SA, Sorra KE, Harris KM. 1999. Slices have more synapses than perfusion-fixed hippocampus from both young and mature rats. J Neurosci 19: 2876-2886.

Knogler LD, Liao M, Drapeau P. 2010. Synaptic scaling and the development of a motor network. J Neurosci 30: 8871-8881.

Leslie KR, Nelson SB, Turrigiano GG. 2001. Postsynaptic depolarization scales quantal amplitude in cortical pyramidal neurons. J Neurosci 21: RC170.

Lu B. 2003. BDNF and activity-dependent synaptic modulation. Learn Mem 10: 86-98.

Maffei A, Nataraj K, Nelson SB, Turrigiano GG. 2006. Potentiation of cortical inhibition by visual deprivation. Nature 443: 81-84.

Maffei A, Turrigiano GG. 2008. Multiple modes of network homeostasis in visual cortical layer 2/3. J Neurosci 28: 4377-4384.

Maffei A, Nelson SB, Turrigiano GG. 2004. Selective reconfiguration of layer 4 visual cortical circuitry by visual deprivation. Nat Neurosci 7: 1353-1359. 
G. Turrigiano

Malenka RC, Bear MF. 2004. LTP and LTD: An embarrassment of riches. Neuron 44: 5-21.

Marder E, Goaillard JM. 2006. Variability, compensation and homeostasis in neuron and network function. Nat Rev Neurosci 7: 563-574.

Marder E, Prinz AA. 2003. Current compensation in neuronal homeostasis. Neuron 37: 2-4.

McCurry CL, Shepherd JD, Tropea D, Wang KH, Bear MF Sur M. 2011. Loss of Arc renders the visual cortex impervious to the effects of sensory experience or deprivation. Nat Neurosci 13: 450-457.

Miller KD, MacKay DJC. 1994. The role of constraints in Hebbian learning. Neural Computation 6: 100-124.

Mrsic-Flogel TD, Hofer SB, Ohki K, Reid RC, Bonhoeffer T, Hubener M. 2007. Homeostatic regulation of eye-specific responses in visual cortex during ocular dominance plasticity. Neuron 54: 961-972.

Nelson SB, Turrigiano GG. 2008. Strength through diversity. Neuron 60: 477-482.

O’Brien RJ, Kamboj S, Ehlers MD, Rosen KR, Fischbach GD, Huganir RL. 1998. Activity-dependent modulation of synaptic AMPA receptor accumulation. Neuron 21: 1067-1078.

Paradis S, Sweeney ST, Davis GW. 2001. Homeostatic control of presynaptic release is triggered by postsynaptic membrane depolarization. Neuron 30: 737-749.

Perez-Otano I, Ehlers MD. 2005. Homeostatic plasticity and NMDA receptor trafficking. Trends Neurosci 28 229-238.

Poon MM, Chen L. 2008. Retinoic acid-gated sequencespecific translational control by RAR $\alpha$. Proc Natl Acad Sci 105: 20303-20308.

Pozo K, Goda Y. 2010. Unraveling mechanisms of homeostatic synaptic plasticity. Neuron 66: 337-351.

Rabinowitch I, Segev I. 2006a. The endurance and selectivity of spatial patterns of long-term potentiation/depression in dendrites under homeostatic synaptic plasticity. J Neurosci 26: 13474-13484.

Rabinowitch I, Segev I. 2006b. The interplay between homeostatic synaptic plasticity and functional dendritic compartments. J Neurophysiol 96: 276-283.

Rich MM, Wenner P. 2007. Sensing and expressing homeostatic synaptic plasticity. Trends Neurosci 30: 119-125.

Rutherford LC, DeWan A, Lauer HM, Turrigiano GG. 1997. Brain-derived neurotrophic factor mediates the activitydependent regulation of inhibition in neocortical cultures. J Neurosci 17: 4527-4535.

Rutherford LC, Nelson SB, Turrigiano GG. 1998. BDNF has opposite effects on the quantal amplitude of pyramidal neuron and interneuron excitatory synapses. Neuron 21: $521-530$.

Seeburg DP, Sheng M. 2008. Activity-induced Polo-like kinase 2 is required for homeostatic plasticity of hippocampal neurons during epileptiform activity. J Neurosci 28: 6583-6591.

Seeburg DP, Feliu-Mojer M, Gaiottino J, Pak DT, Sheng M. 2008. Critical role of CDK5 and Polo-like kinase 2 in homeostatic synaptic plasticity during elevated activity. Neuron 58: 571-583.
Sharpless SK. 1975. Supersensitivity-like phenomena in the central nervous system. Fed Proc 34: 1990-1997.

Shepherd JD, Rumbaugh G, Wu J, Chowdhury S, Plath N, Kuhl D, Huganir RL, Worley PF. 2006. Arc/Arg3.1 mediates homeostatic synaptic scaling of AMPA receptors. Neuron 52: 475-484.

Simon DJ, Madison JM, Conery AL, Thompson-Peer KL, Soskis M, Ruvkun GB, Kaplan JM, Kim JK. 2008. The microRNA miR-1 regulates a MEF-2-dependent retrograde signal at neuromuscular junctions. Cell 133: 903-915.

Stein V, House DR, Bredt DS, Nicoll RA. 2003. Postsynaptic density-95 mimics and occludes hippocampal long-term potentiation and enhances long-term depression. J Neurosci 23: 5503-5506.

Steinberg JP, Takamiya K, Shen Y, Xia J, Rubio ME, Yu S, Jin W, Thomas GM, Linden DJ, Huganir RL. 2006. Targeted in vivo mutations of the AMPA receptor subunit GluR2 and its interacting protein PICK1 eliminate cerebellar long-term depression. Neuron 49: 845-860.

Steinmetz CC, Turrigiano GG. 2010. Tumor necrosis factor- $\alpha$ signaling maintains the ability of cortical synapses to express synaptic scaling. J Neurosci 30: 1468514690.

Stellwagen D, Malenka RC. 2006. Synaptic scaling mediated by glial TNF- $\alpha$. Nature 440: 1054-1059.

Stellwagen D, Beattie EC, Seo JY, Malenka RC. 2005. Differential regulation of AMPA receptor and GABA receptor trafficking by tumor necrosis factor- $\alpha$. J Neurosci 25 : 3219-3228.

Sun Q, Turrigiano GG. 2011. PSD-95 and PSD-93 play critical but distinct roles in synaptic scaling up and down. J Neurosci 31: 6800-6808.

Sun X, Wolf ME. 2009. Nucleus accumbens neurons exhibit synaptic scaling that is occluded by repeated dopamine pre-exposure. Eur J Neurosci 30: 539-550.

Sutton MA, Ito HT, Cressy P, Kempf C, Woo JC, Schuman EM. 2006. Miniature neurotransmission stabilizes synaptic function via tonic suppression of local dendritic protein synthesis. Cell 125: 785-799.

Sutton MA, Taylor AM, Ito HT, Pham A, Schuman EM. 2007. Postsynaptic decoding of neural activity: eEF2 as a biochemical sensor coupling miniature synaptic transmission to local protein synthesis. Neuron 55: 648-661.

Swanwick CC, Murthy NR, Kapur J. 2006. Activity-dependent scaling of GABAergic synapse strength is regulated by brain-derived neurotrophic factor. Molec Cell Neurosci 31: $481-492$.

Terashima A, Pelkey KA, Rah JC, Suh YH, Roche KW, Collingridge GL, McBain CJ, Isaac JT. 2008. An essential role for PICK1 in NMDA receptor-dependent bidirectional synaptic plasticity. Neuron 57: 872-882.

Thiagarajan TC, Lindskog M, Tsien RW. 2005. Adaptation to synaptic inactivity in hippocampal neurons. Neuron 47: 725-737.

Thiagarajan TC, Lindskog M, Malgaroli A, Tsien RW. 2007. LTP and adaptation to inactivity: Overlapping mechanisms and implications for metaplasticity. Neuropharmacology 52: 156-175.

Turrigiano GG. 2008. The self-tuning neuron: Synaptic scaling of excitatory synapses. Cell 135: 422-435. 
Turrigiano G. 2011. Too many cooks? Intrinsic and synaptic homeostatic mechanisms in cortical circuit refinement. Annu Rev Neurosci 34: 89-103.

Turrigiano GG, Nelson SB. 2004. Homeostatic plasticity in the developing nervous system. Nat Rev Neurosci 5: 97-107.

Turrigiano GG, Leslie KR, Desai NS, Rutherford LC, Nelson SB. 1998. Activity-dependent scaling of quantal amplitude in neocortical neurons. Nature 391: 892-896.

Vale C, Sanes DH. 2002. The effect of bilateral deafness on excitatory and inhibitory synaptic strength in the inferior colliculus. Eur J Neurosci 16: 2394-2404.

Volk L, Kim CH, Takamiya K, Yu Y, Huganir RL. 2010. Developmental regulation of protein interacting with $\mathrm{C}$ kinase 1 (PICK1) function in hippocampal synaptic plasticity and learning. Proc Natl Acad Sci 107: 21784-21789.

Watt AJ, van Rossum MC, MacLeod KM, Nelson SB, Turrigiano GG. 2000. Activity coregulates quantal AMPA and
NMDA currents at neocortical synapses. Neuron 26: 659-670.

Wierenga CJ, Ibata K, Turrigiano GG. 2005. Postsynaptic expression of homeostatic plasticity at neocortical synapses. J Neurosci 25: 2895-2905.

Wierenga CJ, Walsh MF, Turrigiano GG. 2006. Temporal regulation of the expression locus of homeostatic plasticity. J Neurophysiol 96: 2127-2133.

Xu W, Schluter OM, Steiner P, Czervionke BL, Sabatini B, Malenka RC. 2008. Molecular dissociation of the role of PSD-95 in regulating synaptic strength and LTD. Neuron 57: $248-262$.

Yu LM, Goda Y. 2009. Dendritic signalling and homeostatic adaptation. Curr Opin Neurobiol 19: 327-335.

Zhang W, Linden D.J.. 2003. The other side of the engram: Experience-driven changes in neuronal intrinsic excitability. Nat Rev Neurosci 4: 885-900. 


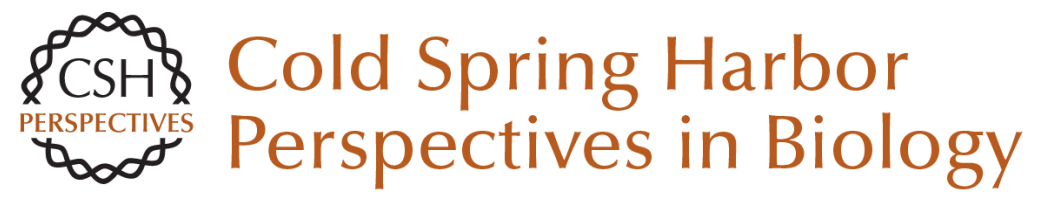

\section{Homeostatic Synaptic Plasticity: Local and Global Mechanisms for Stabilizing Neuronal Function}

Gina Turrigiano

Cold Spring Harb Perspect Biol 2012; doi: 10.1101/cshperspect.a005736 originally published online November 15, 2011

Subject Collection The Synapse

Studying Signal Transduction in Single Dendritic

Spines

Ryohei Yasuda

Synaptic Vesicle Pools and Dynamics

AbdulRasheed A. Alabi and Richard W. Tsien

Synapses and Memory Storage

Mark Mayford, Steven A. Siegelbaum and Eric $R$. Kandel

Synapses and Alzheimer's Disease

Morgan Sheng, Bernardo L. Sabatini and Thomas C. Südhof

Synaptic Cell Adhesion

Markus Missler, Thomas C. Südhof and Thomas Biederer

Synaptic Dysfunction in Neurodevelopmental

Disorders Associated with Autism and Intellectual

Disabilities

Huda Y. Zoghbi and Mark F. Bear

The Postsynaptic Organization of Synapses Morgan Sheng and Eunjoon Kim

Presynaptic LTP and LTD of Excitatory and Inhibitory Synapses

Pablo E. Castillo
Synaptic Vesicle Endocytosis

Yasunori Saheki and Pietro De Camilli

Short-Term Presynaptic Plasticity Wade G. Regehr

NMDA Receptor-Dependent Long-Term

Potentiation and Long-Term Depression

(LTP/LTD)

Christian Lüscher and Robert C. Malenka

Ultrastructure of Synapses in the Mammalian

Brain

Kristen M. Harris and Richard J. Weinberg

Calcium Signaling in Dendritic Spines

Michael J. Higley and Bernardo L. Sabatini

Synaptic Neurotransmitter-Gated Receptors

Trevor G. Smart and Pierre Paoletti

Synaptic Vesicle Exocytosis

Thomas C. Südhof and Josep Rizo

Vesicular and Plasma Membrane Transporters for Neurotransmitters

Randy D. Blakely and Robert H. Edwards

For additional articles in this collection, see http://cshperspectives.cshlp.org/cgi/collection/

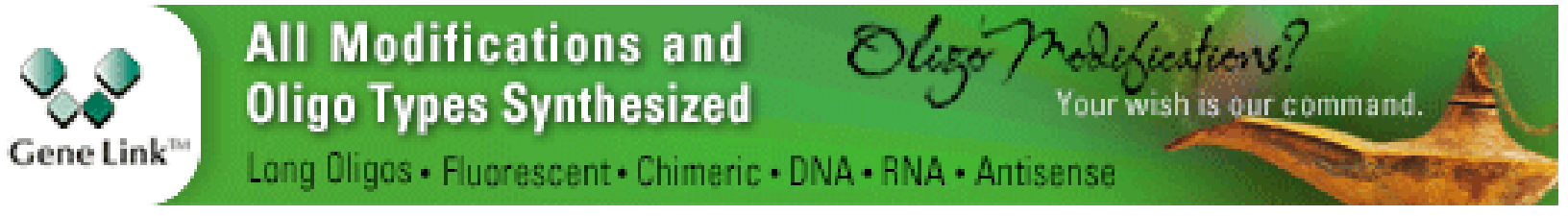

Copyright @ 2012 Cold Spring Harbor Laboratory Press; all rights reserved 